ISSN 0103-5150

Fisioter. Mov., Curitiba, v. 26, n. 3, p. 587-594, jul./set. 2013

Licenciado sob uma Licença Creative Commons

\title{
Análise da musculatura estabilizadora lombopélvica em jovens com e sem dor lombar
}

\author{
Lumbopelvic stabilization musculature analysis in \\ young subjects with and without low back pain
}

\section{Jarbas Melo Filho $^{[a]}$, Berlis Ribeiro dos Santos Menossi ${ }^{[b]}$, Cássio Preis ${ }^{[c]}$, Luiz Bertassoni Neto ${ }^{[\mathrm{d}]}$, Antônio Stabelini Neto ${ }^{[e]}$}

[a] Fisioterapeuta, aluno de Pós-Graduação em Fisioterapia Ortopédica, Traumatológica e Desportiva pela Pontifícia Universidade Católica do Paraná (PUCPR) e em Fisiologia do Exercício Físico pela Universidade Estadual do Norte do Paraná (Uenp), Jacarezinho, PR - Brasil, e-mail: jarbasmf@hotmail.com

[b] Fisioterapeuta, educadora física, mestre em Educação Física pela Universidade Estadual de Campinas (Unicamp), com aprimoramento em Medicina da Reabilitação na Universidade de Liège - Bélgica, professora da Universidade Estadual do Norte do Paraná (Uenp), Jacarezinho, PR - Brasil, e-mail: berlis@sementesorria.com.br

[c] Fisioterapeuta, mestre em Tecnologia em Saúde pela Pontifícia Universidade Católica do Paraná (PUCPR), professor e responsável pelo Centro de Dinamometria Isocinética e Baropodometria da PUCPR, Curitiba, PR - Brasil, e-mail: cassio.preis@pucpr.br

[d] Fisioterapeuta, educador físico, mestre em Educação Superior pela Pontifícia Universidade Católica do Paraná (PUCPR), especialista em Fisioterapia Esportiva pela Sociedade Nacional de Fisioterapia esportiva, especialista em Fisioterapia Tráumato-Ortopédica Funcional pelo Conselho Federal de Fisioterapia e Terapia Ocupacional, Curitiba, PR - Brasil, e-mail: luizbertassoni@yahoo.com.br

[e] Educador físico, especialista em Fisiologia do Exercício pela Universidade Federal do Paraná (UFPR), doutorando em Educação Física na mesma instituição, Jacarezinho, PR - Brasil, e-mail: asneto@uenp.edu.br

\section{Resumo}

Introdução: A capacidade de controle da musculatura profunda local em indivíduos com dor lombar tende a estar diminuída. Objetivo: Analisar e comparar o comportamento da musculatura estabilizadora lombopélvica em jovens com e sem dor lombar. Materiais e métodos: A pesquisa do tipo transversal quantitativa teve aplicação de avaliações para estabilidade lombopélvica por meio dos testes de Resistência Estática do Tronco, Resistência das Costas de Sorenson e Teste de Side Bridge (ponte lateral), questionário funcional lombar de Roland-Morris; a escala de dor foi avaliada com uso da escala análoga visual da dor em adultos 
jovens da Universidade Estadual do Norte do Paraná (UENP), Centro de Ciências da Saúde, Jacarezinho (PR). Resultados: A amostra do trabalho foi composta de 108 indivíduos, 17 do gênero masculino e 91 do gênero feminino, apresentando médias e desvios padrão respectivamente de: idade 19,44 $\pm 1,73$ anos, estatura $167 \pm 8$ $\mathrm{cm}$, peso $60,48 \pm 11 \mathrm{~kg}$ e índice de massa corpórea (IMC) $21,70 \pm 2,80 \mathrm{~kg} / \mathrm{m}^{2}$. Dos participantes, $20 \%$ apresentaram dor lombar. Na comparação entre grupos de indivíduos com dor lombar e sem dor mediante testes de estabilidade lombopélvica, não se observou diferenças significativas, $\mathrm{p}>0,05$ para todos os testes, mesmo com divisão por gênero, onde o percentual dos indivíduos com dor foi de 18,68\% no grupo feminino e de $29,41 \%$ no masculino. Conclusão: Constatou-se que os adultos jovens com dor lombar não se apresentaram com a musculatura estabilizadora lombopélvica significativamente diferente quando comparados com os sem dor.

Palavras-chave: Estabilização. Dor lombar. Adulto jovem.

\begin{abstract}
Introduction: Individuals with low back pain seem to impair the ability to control the muscles deep site. Objective: To analyze and compare the behavior of the lumbopelvic stabilizing musculature in young adult with and without low back pain. Materials and methods: The study was cross-sectional quantitative application of assessments for lumbar-pelvic stability, through tests Trunk Static Resistance, Resistance of the Coasts of Sorenson and Side Bridge Test, functional questionnaire lumbar Roland Morris and pain scale by visual analogue scale of pain in young State University North of Paraná (UENP), Center for Health Sciences, Jacarezinho, Parana State, Brazil. Results: The study sample consisted of 108 individuals, 17 males and 91 females, with mean and standard deviation respectively: age $19.44 \pm 1.73$ years, height $167 \pm 8 \mathrm{~cm}$, weight $60.48 \pm 11 \mathrm{~kg}$ and body mass index (BMI) $21.70 \pm 2.80 \mathrm{~kg} / \mathrm{m}^{2} .20 \%$ of participants had low back pain. When comparing groups of individuals with low back pain and pain through stability testing lumbopelvic not observed significant differences, $p>0.05$ for all tests, even dividing by gender, where the percentage of individuals with pain was $18.68 \%$ among females and $29.41 \%$ among males. Conclusion: It was found that young adults with low back pain were not presented with the lumbopelvic stabilizing musculature significantly different when compared with those without pain.
\end{abstract}

Keywords: Stabilization. Low back pain. Young adult.

\section{Introdução}

O termo "estabilização" refere-se ao controle mecânico articular, o qual se refere à ação dos músculos sobre os limitadores e controladores do movimento e na prevenção de danos a ligamentos e cápsulas (1).

A estabilidade da coluna vertebral depende da integração de três sistemas: sistema passivo (composto pelos corpos vertebrais, articulações facetárias, cápsulas articulares, ligamentos espinhais e discos intervertebrais); sistema ativo (constituído de músculos e tendões); e sistema neural (sistema nervoso central e periférico). Esses subsistemas, sob condições normais, garantem a estabilidade estática e dinâmica da coluna (1). Além de ser um componente importante para prover uma base sólida e exercer ou resistir às forças, o aperfeiçoamento da estabilidade segmentar e do controle neuromuscular do tronco pode proporcionar melhores condições biomecânicas (2).

Indivíduos com dor lombar parecem diminuir a capacidade de controle da musculatura profunda local. França et al. (3) acrescenta que a instabilidade é resultado de uma lesão tecidual que torna o segmento mais fraco ou insuficientemente resistente, ou seja, com fraco controle muscular; por isso é sugerida como causa de desordens funcionais, tensões e dor.

Teoricamente, músculos fracos atingem a condição isquêmica e de fadiga mais facilmente que músculos fortes, aumentando as probabilidades de lesões e dificultando o alinhamento adequado da coluna. Em geral, pessoas mais fracas apresentam a musculatura do abdômen enfraquecida e a musculatura da coluna menos flexível, por esse motivo, necessitam de mais esforços para realizar determinadas tarefas, estão 
também mais expostas a lesões, são pouco flexíveis, e têm dificuldades para manter diferentes posturas (4).

Estudos também apontam que pacientes com dor lombar crônica apresentam deficit de força do tronco e a perda de força dos músculos extensores é muito maior do que dos flexores (5).

Kavcic et al.(6) sugerem que qualquer disfunção lombar acarreta dificuldade no recrutamento dos músculos estabilizadores para a conservação da estabilidade da coluna. Sendo assim, numa disfunção musculoesquelética, os músculos ao redor da articulação lesada são afetados podendo gerar fraqueza e consequente atrofia muscular, o que aumenta o quadro de instabilidade.

Com a disfunção local ocorre substituição compensatória de músculos globais, que pode ser explicada pela tentativa do sistema neural em manter a estabilidade por meio da solicitação dos músculos globais (7).

Os músculos profundos - transverso do abdômen e multífidos lombar - trabalham para manter a estabilidade da coluna vertebral e tronco, com ou sem movimentos de membros, e por isso recebe muitos tipos de terapias físicas. 0 fortalecimento desses músculos com o trabalho combinado da musculatura abdominal e extremidades superiores e inferiores auxiliam na prevenção e reabilitação de desordens musculoesqueléticas (8).

Segundo a Organização Mundial de Saúde (OMS), cerca de $80 \%$ dos adultos desenvolverão durante a vida pelo menos uma crise de dor lombar, e $90 \%$ destes apresentarão mais de um episódio, existindo evidências de que a musculatura profunda do abdômen, especialmente o transverso abdominal e multífidos, é afetada na lombalgia e instabilidade segmentar (7, $8,9)$. Por essa razão, a participação dos músculos da parede abdominal como agentes estabilizadores da coluna e a eficácia dos exercícios de estabilização segmentar na promoção de tal estabilidade têm sido enfatizadas $(3,8,7)$.

Sendo assim, o estudo teve o objetivo de analisar o comportamento da musculatura estabilizadora lombopélvica em jovens com e sem dor lombar por meio de testes de estabilidade, questionário funcional lombar e escala de dor.

\section{Materiais e métodos}

O trabalho do tipo transversal quantitativo (10) valeu-se de avaliação da estabilidade lombopélvica, questionário funcional lombar e escala de dor. A amostra foi escolhida por conveniência, tendo como voluntários do estudo acadêmicos do Centro de Ciências da Saúde (CCS) da Universidade Estadual do Norte do Paraná (Uenp) do ano de 2010 com idade entre 18 e 30 anos.

Os fatores de inclusão foram: faixa etária entre 18 e 30 anos; acadêmico do curso de Fisioterapia do CCS-UENP, não praticante de atividade física regular. Os fatores de exclusão foram: presença de lesão osteomioligamentar que impossibilitasse a realização dos testes de estabilidade lombopélvica, gestantes, uso de medicação analgésica e/ou anti-inflamatória e/ou não ter completado a bateria dos testes propostos na pesquisa.

Para início da pesquisa o projeto foi apresentado ao Comitê de Ética em Pesquisa da Pontifícia Universidade Católica do Paraná (PUCPR) e após a sua aprovação pelo protocolo n. 5787 de 15/10/2010 foi realizada a aplicação prática experimental. Os avaliados assinaram um Termo de Consentimento Livre e Esclarecido, no qual constava toda a execução da pesquisa, autorizando a utilização dos dados coletados.

Utilizou-se para coleta dos dados antropométricos o estadiômetro da marca Cardiomed e a balança da marca Plenna.

Para avaliar a estabilidade lombopélvica dos indivíduos, foram aplicados os seguintes testes:

a) Teste de Resistência Estática do Tronco (11) - 0 avaliado posiciona-se sentado, com quadris e joelhos fletidos a $90^{\circ}$, braços dobrados e cruzados no tórax e os pés fixados pelo avaliador. Com o tronco em uma angulação de $60^{\circ}, \mathrm{o}$ avaliado mantém a postura isométrica pelo maior tempo possível.

b) Teste de Resistência das costas de Sorenson (11-13) - 0 avaliado posiciona-se em decúbito ventral com a crista ilíaca anterossuperior no final da maca de exame. 0 tronco deve estar estendido fora da maca e os braços ao lado do tronco com fixação das coxas e tornozelos. $\mathrm{O}$ avaliado mantém uma posição neutra (não hiperestendida) por maior tempo possível; e o teste interrompido após quatro minutos.

c) Teste de Side Bridge (ponte lateral) (1113). 0 avaliado posiciona-se em decúbito lateral sustentando sua pelve apoiado nos pés 
e antebraço inferior. 0 pé superior é colocado na frente do inferior. 0 braço superior é colocado ao lado do tronco. A posição é mantida estaticamente pelo maior tempo.

Os voluntários foram divididos em dois grupos, os que apresentavam dor lombar anterior aos testes de estabilidade lombopélvica (Grupo 1) e sem presença de dor lombar (Grupo 2).

Como formas de quantificar a função e dor dos participantes que apresentassem dor lombar foram utilizados o questionário de Roland-Morris e a escala análoga visual da dor (EVA) em fichas individuais na forma de autoavaliação. 0 questionário Roland-Morris é composto por 24 questões relevantes para descrição da funcionalidade de pacientes com dor lombar; é atribuído um ponto a cada frase assinalada, sendo o resultado a somatória desses pontos. Quanto maior a pontuação final, maior será a incapacidade do indivíduo. A pontuação mínima é zero e representa nenhum impacto da dor sobre a pessoa, o valor máximo é 24 , indicativo de incapacidade funcional total. Não há direcionamento de atenção aos aspectos psicossociais englobados na lombalgia. A escala visual análoga da dor é atribuída em uma régua de $0 \mathrm{a}$ 10; zero representa nenhuma dor, e dez, máxima dor sentida pelo indivíduo (14-16).

Os dados obtidos foram analisados em tabelas do programa Microsoft Excel e testados estatisticamente utilizando-se o programa SPSS for Windows 13.0.

\section{Resultados}

A amostra do trabalho foi de 108 indivíduos, 17 do gênero masculino e 91 do gênero feminino, com médias e desvios padrão de: idade, 19,44 $\pm 1,73$ anos, estatura, $167 \pm 8 \mathrm{~cm}$, peso, $60,48 \pm 11 \mathrm{~kg}$; e índice de massa corpórea (IMC), 21,70 $\pm 2,80 \mathrm{~kg} / \mathrm{m}^{2}$ (17).

0 Gráfico 1 mostra a porcentagem de indivíduos que apresentaram dor lombar comparado ao grupo sem dor.

Na aplicação do questionário de Roland-Morris, observou-se que os respondentes tiveram um escore médio de 3,77 , o que evidencia um pequeno impacto sobre a funcionalidade da coluna lombar desses indivíduos. Para a escala análoga visual da dor, a pontuação média foi de 3,32, também apresentando baixa intensidade.

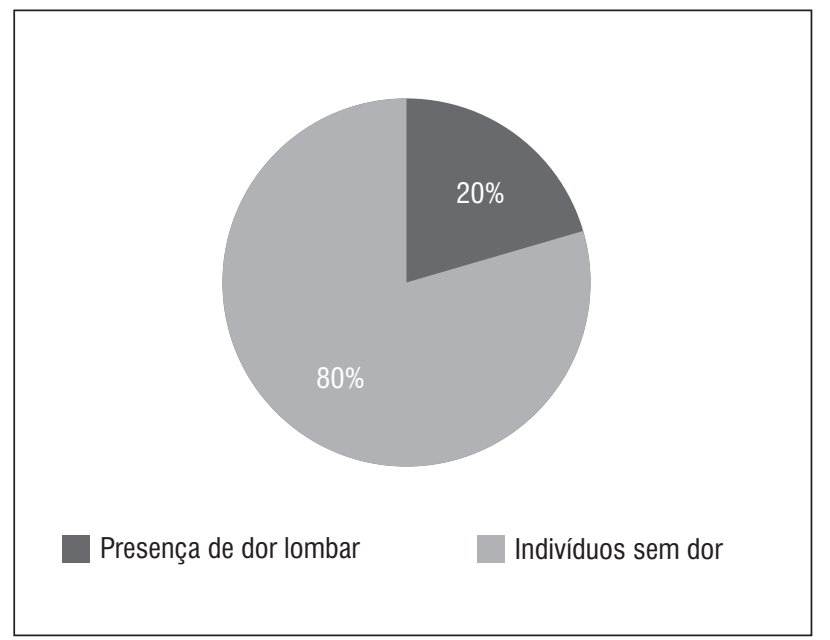

Gráfico 1 - Análise percentual dos indivíduos em relação à presença de dor lombar

Fonte: Dados da pesquisa.

Para testar a normalidade da amostra, empregou-se o teste de Kolmogorov-Smirnov, apresentando um resultado não significativo, $\mathrm{p}<0,05$ para todas as variáveis, aplicando a partir desse resultado o teste de Mann-Whitney U.

O Gráfico 2 apresenta a comparação dos testes de estabilidade lombopélvica entre grupos, divididos em Grupo 1 (dor lombar) e Grupo 2 (sem dor), não apresentando significância, $p>0,05$, para todos os testes.

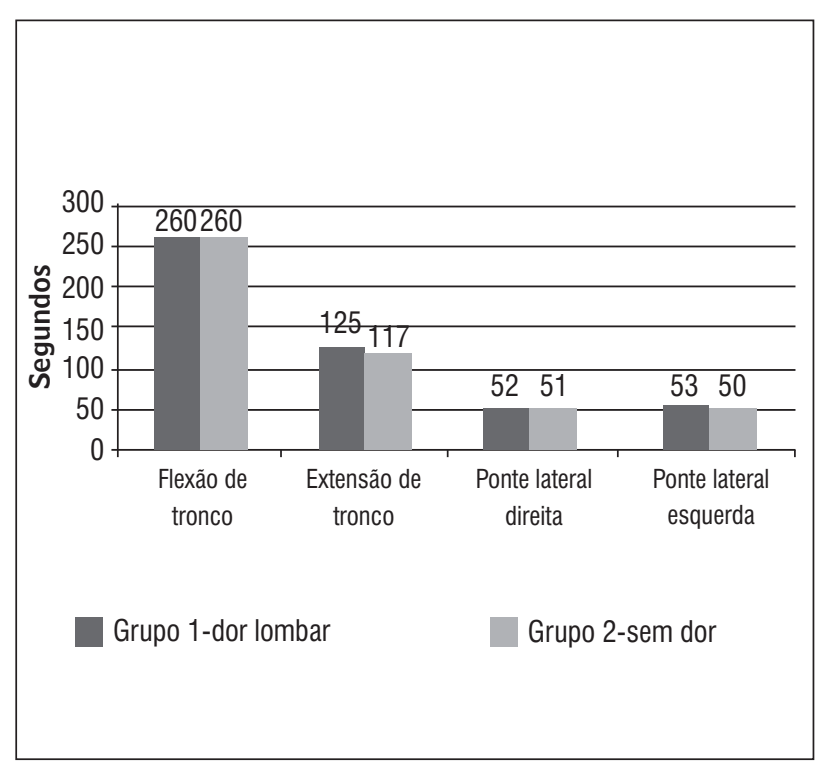

Gráfico 2 - Valores em médias dos testes de estabilidade lombopélvica na comparação por grupos

Fonte: Dados da pesquisa. 
A amostra também foi separada por gênero e comparada em relação aos testes de estabilidade lombopélvica entre grupos com e sem dor lombar, não apresentando significância, $p>0,05$, para todos os testes tanto para o feminino quanto para o masculino. A porcentagem dos indivíduos com dor lombar foi de $18,68 \%$ no grupo feminino e $29,41 \%$ no masculino.

0 Gráfico 3 apresenta a comparação entre grupos, com e sem dor lombar, dos testes de estabilidade do

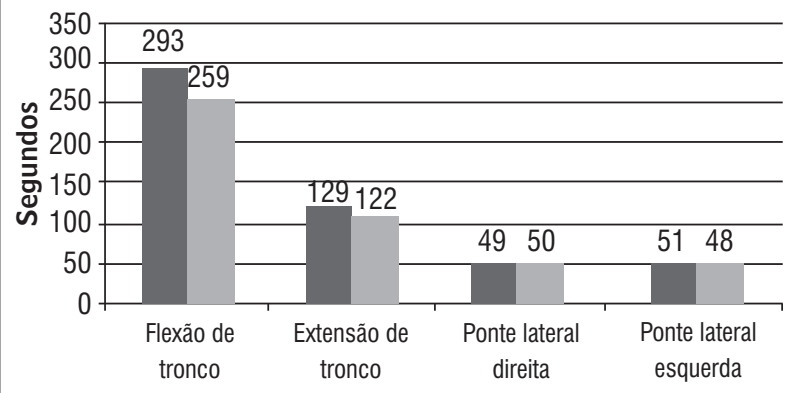

Grupo 1 - dor lombar

Grupo 2 - sem dor

Gráfico 3 - Valores em médias dos testes de estabilidade Iombopélvica na comparação entre grupos para sujeitos do gênero feminino

Fonte: Dados da pesquisa.

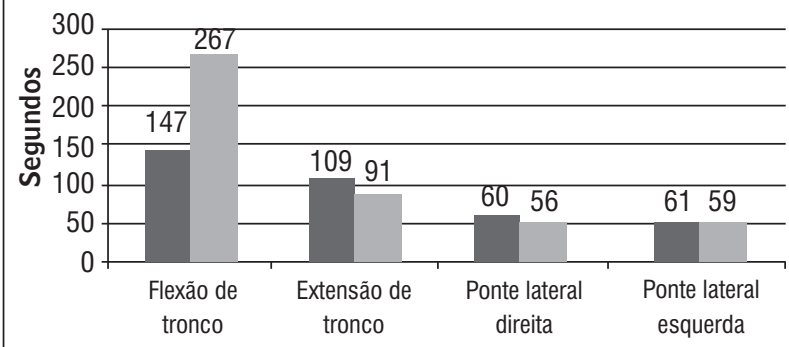

Grupo 1 - dor lombar

Grupo 2 - sem dor

Gráfico 4 - Valores em médias dos testes de estabilidade lombopélvica na comparação entre grupos para sujeitos do gênero masculino

Fonte: Dados da pesquisa. gênero feminino; e no Gráfico 4, apresenta-se a comparação para o gênero masculino.

\section{Discussão}

Sabe-se que a instabilidade do segmento lombar representa um dos fatores de maior incidência em pacientes com dor lombar crônica (1). Há evidências crescentes de que os músculos profundos da parede abdominal e os multífidos lombares são frequentemente afetados em sujeitos que apresentam dores crônicas, agudas ou instabilidade lombar (18-21).

Medir a habilidade motora dos músculos profundos é uma tarefa complexa, pois esses músculos localizam-se internamente, tornando sua ação contrátil difícil de ser visualizada no nível da superfície. Tais músculos apresentam uma contração leve e isométrica, que são executadas independentes dos músculos globais. A ação dos músculos profundos pode ser confundida por possíveis atividades aumentadas ou compensatórias dos músculos globais, porém, sua ação é específica e deve ser minuciosamente isolada (22).

Os estudos mais recentes utilizam a eletromiografia como principal recurso para avaliação da musculatura profunda, obtendo uma análise precisa da atividade muscular, muitas vezes com associação com os testes de resistência isométrica utilizados neste trabalho (22-25).

Também a dinamometria isocinética do tronco é, cada vez mais, utilizada como padrão-ouro de avaliação para flexores e extensores do tronco, a fim de averiguar entre outros fatores o equilíbrio muscular entre agonistas e antagonistas. No entanto, esse procedimento apresenta um inconveniente relativo a seu alto custo (26); por essa razão, mostra-se como limitação do estudo na escolha da avaliação muscular.

Nesta pesquisa não se pode afirmar que os indivíduos com dor lombar apresentam a musculatura estabilizadora lombopélvica diferenciada dos indivíduos sem dor nos testes de resistência estática do tronco, resistência das costas de Sorenson e ponte lateral. Possivelmente, isso se deva ao fato de a dor e a disfunção não serem de grande impacto na população.

Em um estudo com indivíduos saudáveis com idade média de 22 anos, observou-se que não há diferença significativa entre o teste de ponte lateral direita $\mathrm{e}$ esquerda, o que corrobora com a pesquisa atual (27). 
Quando ocorre dor em um segmento, parece haver uma substituição compensatória da ação dos músculos do sistema global em relação ao sistema muscular local. Esta pode ser uma ação do sistema nervoso central na tentativa de suprir a necessidade de manter a estabilidade diante de forças atuantes sobre esse segmento (28).

A hipótese de instabilidade assume uma relação entre movimento intersegmentar anormal e dores lombares. A contribuição para essa hipótese é que a diminuição do movimento intersegmentar possa resultar em uma consequente diminuição de dor no paciente com lombalgia. Na verdade, isso serve de base para o próprio tratamento de pacientes com dores lombares, envolvendo assim, fortalecimentos, alongamentos e controle motor dos músculos $(1,29)$.

A natureza direcional de instabilidade fundamenta-se no mecanismo de lesão, local resultante de dano ao tecido. 0 local da lesão dominante no segmento de movimento determina o padrão de instabilidade manifestado. Como os movimentos na coluna lombar são tridimensionais e envolvem movimentos combinados, as lesões teciduais podem resultar provavelmente de deficiências orgânicas associadas a movimentos com mais de uma direção (1).

A estabilidade lombopélvica cria várias vantagens por integração dos segmentos proximal e distal gerando e controlando forças para maximizar a função; além disso, está envolvida com quase todas as atividades de extremidades $(22,30)$.

Várias pesquisas constataram a eficácia da estabilização segmentar nas lombalgias e, principalmente, na prevenção de sua recidiva, por atuar diretamente no controle motor, devolvendo a função protetora dos músculos profundos $(3,8,26,31,32)$.

A estabilidade corporal, responsável pelo alinhamento do corpo, é diretamente ligada ao controle do Sistema Nervoso Central pela resposta sensorial das estruturas osteoligamentares e pelo controle da musculatura ativa (11). Logo, qualquer disfunção em um desses fatores promove instabilidade. 0 corpo tende a compensar essa instabilidade de alguma forma, causando desequilíbrio entre músculos e dor.

Contudo, fazem-se necessárias atividades preventivas e/ou reabilitativas para melhora do padrão antecipatório para a estabilidade lombopélvica. Um desequilíbrio na força muscular do tronco pode ser um fator de risco para dor lombar, segundo estudo prospectivo de cinco anos realizado por Lee et al. (33).
Como o estudo foi realizado com população por conveniência e em apenas uma avaliação, não houve perda amostral, o que contribuiu para a formação de uma amostra considerável.

\section{Conclusão}

Em relação à resistência da musculatura estabilizadora lombopélvica, este estudo demonstrou que os indivíduos que apresentaram dor lombar não se diferenciaram significantemente daqueles que não apresentaram dor, mesmo com divisão por gênero. 0 fato de a dor e da disfunção não terem sido de grande impacto nos indivíduos que a apresentaram pode ter contribuído para esse resultado. Sugere-se que novos estudos sejam realizados em relação a diferentes populações com ênfase sobre as formas de avaliação da musculatura estabilizadora lombopélvica.

\section{Referências}

1. Panjabi MM. Clinical spinal instability and low back pain. J Electromyogr Kinesio. 2003;13(4):371-9.

2. Carvalho A, Assini TCKA. Aprimoramento da capacidade funcional de idosos submetidos a uma intervenção por isostretching. Rev Bras Fisioter. 2008;12(4):268-73.

3. França FJR, Burke TN, Claret DC, Marques AP. Estabilização segmentar da coluna lombar nas lombalgias: uma revisão bibliográfica e um programa de exercícios. Fisioter Pesqui. 2008;15(2):200-6.

4. Toscano JJO, Egypto EP. A influência do sedentarismo na prevalência de lombalgia. Rev Bras Med Esporte. 2001;7(4):132-7.

5. Cohen I, Rainville J. Agressive exercise as treatment for chronic low back pain. Sports Medicine. 2002;32(1):75-82.

6. Kavcic N, Grenier S, McGill SM. Quantifying tissue loads and spine stabilization while performing commonly prescribed low back stabilization exercises. Spine. 2004;29(20):2319-29.

7. Gouveia KMC; Gouveia EC. O músculo transverso abdominal e sua função de estabilização da coluna lombar. Fisioter Mov. 2008;21(3):45-50. 
8. Reinehr FB, Carpes FP, Mota CB. Influência do treinamento de estabilização central sobre a dor e estabilidade lombar. Fisioter Mov. 2008;21(1):123-9.

9. Costa LOP, Costa LCM, Cançado RL, Oliveira WM, Ferreira PH. Confiabilidade do teste palpatório e da unidade de biofeedback pressórico na ativação do músculo transverso abdominal em indivíduos normais. Acta Fisiatr. 2004;11(3):101-5

10. Curi PR. Metodologia e análise da pesquisa em Ciências Biológicas. 2. ed. Botucatu: Tipomic; 1998.

11. Liebenson C. Spinal stabilization - an update. Part 2 - functional assessment. J Bodyw Mov Ther. 2004;8(3):199-210.

12. Jassi FJ. Análise do comportamento eletromiográfico dos músculos estabilizadores primários e a relação da capacidade física funcional de indivíduos assintomáticos. [dissertação]. Presidente Prudente: Faculdade de Ciências e Tecnologia, Universidade Estadual Paulista; 2010.

13. Cox JM. Dor lombar: mecanismo, diagnóstico e tratamento. 6. ed. São Paulo: Manole; 2002.

14. Nusbaum L, Natour J, Ferraz MB, Goldenberg J. Translation, adaptation and validation of the Roland-Morris questionnaire - Brazil Roland-Morris. Braz J Med Biol Res. 2001;34(2):203-10.

15. Tsukimkoto GR, Riberto M, Brito CA, Battistela LR. Avaliação longitudinal da Escola de Postura para dor lombar crônica através da aplicação dos questionários Roland Morris e Short Form Health Survey (SF-36). Acta Fisiatr. 2006;13(2):63-9.

16. Agne JE. Eletrotermoterapia: teoria e prática. Santa Maria: Orium; 2005.

17. Portal da Saúde. Peso ideal. [acesso 20 ago 2010]. Disponível em: http://portal.saude.gov.br/portal/ saude/visualizar_texto.cfm?idtxt=29922\&janela=1

18. O'Sullivan SB, Schmtz TJ. Fisioterapia: avaliação e tratamento. 2. ed. São Paulo: Manole; 2004.

19. Hogdes PW, Richardson CA. Inefficient muscular stabilization of the lumbar spine associated with low back pain. A motor control evaluation of the transversus abdominis. Spine. 1996;21(22):2640-50.
20. Hides JA, Richardson CA, Jull GA. Multifidus muscle recovery is not automatic after resolution of acute, first-episode low back pain. Spine. 1996;21(23):2763-69.

21. Hogdes PW, Richardson CA. Delayed postural contraction of the transversus abdominais in low back pain associated with movement of the lower limb. J Spinal Disord. 1998;11(1):45-56.

22. Marshall PW, Murphy BA. Core stability exercises on and off a Swiss ball. Arch Phys Med Rehabil. 2005;86:242-9.

23. Chok B, Lee R, Latimer J, Tan SB. Endurance training of the trunk extensor muscles in people with subacute low back pain. Phys Ther. 1999;79(11):1032-42.

24. O'Sulivan PB. Lumbar segmental 'instability': clinical presentation and specific stabilizing exercise management. Man Ther. 2001;5(1):2-12.

25. Willson DJ; Dougherty CP, Ireland ML, Davis IM. Core stability and its relationship to lower extremity function and injury. J Am Acad Orthop Surg. 2005;13(5):316-25.

26. Melo J Filho, Eduardo FMC; Moser AD. Estabilização lombopélvica: evidências em avaliação e tratamento. Fisioter Bras. 2011;12(6):467-76.

27. McGill SM, Childs A, Liebenson C. Endurance times for stabilization exercises: clinical targets for testing and training from a normal database. Arch Phys Med Rehabil. 1999;80:941-4.

28. Gibson J, Mccarron T. Feedforward muscle activity: an investigation into the onset and activity of internal oblique during two functional reaching tasks. J Bodyw Mov Ther. 2004;8(2):104-13.

29. Richardson C, Jull G, Hodges P, Hides J. Therapeutic exercise for spinal segmental stabilization in low back pain: scientific basis and clinical approach. Edinburgh: Churchill Livingstone; 1999.

30. Hodges PW; Richardson CA. Feedforward contraction of transversus abdominis is not influenced by the direction of arm movement. Exp Brain Res. 1997;114:362-70.

31. Norris CM. Spinal stabilization. 1 - Active lumbar stabilization - concepts. Physiot. 1999;81(2):61-3. 
32. Richardson CA, Toppenberg R, Jull GA. An initial evaluation of eight abdominal exercises for their ability to provide stabilization for the lumbar spine. Aust J Physiother. 1990;36:6-11.

33. Lee J, Hoshino Y, Nakamura K, Kariya Y, Saita K, Ito K. Runk muscle weakness as a risk factor for low back pain. A 5-year prospective study. Spine. 1999;24(1):54-7.

Recebido: $15 / 07 / 2012$

Received: 07/15/2012

Aprovado: 25/03/2012

Approved: 03/25/2012 